\title{
Col9a2 gene deletion accelerates the degeneration of intervertebral discs
}

\author{
HUIHUI XU ${ }^{1,2^{*}}$, RUI DONG ${ }^{1,3^{*}}$, QINGHE ZENG ${ }^{1,2^{*}}$, LIANG FANG $^{1,2}$, QINWEN GE ${ }^{1,2}$, CHENJIE XIA $^{1,2}$, \\ PENG ZHANG ${ }^{1,2}$, SHUAIJIE LV ${ }^{1-3}$, ZHEN ZOU $^{1,2}$, PINGER WANG ${ }^{1,2}$, JU LI $^{1-3}$, HONGFENG RUAN $^{1,2}$, \\ SONGFENG HU ${ }^{4}$, CHENGLIANG WU ${ }^{1,2}$, HONGTING JIN ${ }^{1,2}$ and PEIJIAN TONG ${ }^{1-3}$ \\ ${ }^{1}$ The First College of Clinical Medicine, Zhejiang Chinese Medical University; ${ }^{2}$ Institute of Orthopaedics and \\ Traumatology of Zhejiang Province, Hangzhou, Zhejiang 310053; ${ }^{3}$ Department of Orthopaedic Surgery, \\ The First Affiliated Hospital of Zhejiang Chinese Medical University, Hangzhou, Zhejiang 310006; ${ }^{4}$ Department of \\ Orthopaedics and Traumatology, Shaoxing Hospital of Traditional Chinese Medicine, Shaoxing, Zhejiang 312000, P.R. China
}

Received August 29, 2021; Accepted December 22, 2021

DOI: $10.3892 / \mathrm{etm} .2022 .11130$

\begin{abstract}
As an essential component of the extracellular matrix (ECM) in cartilage, the $\alpha 2$ chain of type IX collagen (Co19a2), has been implicated in human intervertebral disc degeneration (IVDD). However, the precise role of the Col9a2 gene in the pathogenesis of IVDD has remained elusive. In the present study, the spines of Col9a2-deficient $\left(\mathrm{Col} 9 \mathrm{a}^{-/}\right)$mice were systematically analyzed and compared with wild-type control mice using micro-CT $(\mu \mathrm{CT})$, histomorphology, immunofluorescence, immunohistochemistry and reverse transcription-quantitative PCR (RT-qPCR). $\mu \mathrm{CT}$ analysis revealed that endplate (EP) osteochondral remodeling in the Col9a $2^{-/}$group was accompanied by a significant increase in EP porosity. Likewise, histopathological staining at 12 weeks revealed that the Col9a $2^{-/-}$mice exhibited a marked early-stage IVDD phenotype, including EP sclerosis, calcification and annulus fibrosus rupture. The immunofluorescence results indicated that Col9a2 was extensively expressed in the IVDs, whereas it was barely detectable in Col9a $2^{-/-}$mice. Immunohistochemical and RT-qPCR analyses demonstrated that the expression levels of Col2al and Aggrecan in the IVDs of Col9a $2^{-/-}$mice were significantly decreased. In addition, the levels of Mmp13, ADAM metallopeptidase with thrombospondin type 1 motif 5, Col10a1 and Runx family transcription factor 2 were significantly elevated. These results suggested
\end{abstract}

Correspondence to: Dr Hongting Jin or Dr Peijian Tong, The First College of Clinical Medicine, Zhejiang Chinese Medical University, 548 Binwen Road, Hangzhou, Zhejiang 310053, P.R. China

E-mail: hongtingjin@163.com

E-mail: peijiantongzjtcm@163.com

*Contributed equally

Key words: Co19a2, endplate, calcification, ossification, intervertebral disc degeneration that deletion of the Co19a2 gene led to osteochondral remodeling of cartilage EP and suppressed ECM synthesis, accelerating matrix degradation and chondrocyte hypertrophy in the IVD tissue.

\section{Introduction}

Intervertebral disc degeneration (IVDD) is one of the most common musculoskeletal disorders observed in clinical practice. Substantial evidence has demonstrated that lower back pain and spinal cord compression nerve pain are prevalent clinical and public health concerns caused by IVDD $(1,2)$. The etiology of IVDD is multifactorial, including aging, genetic predisposition, high mechanical stress, metabolic disorders, insufficient nutrition and neurogenic inflammation $(3,4)$. At present, the therapeutic strategies mainly focus on pain relief through injections, physical therapy and activity modification or surgical interventions, such as disc decompression, spinal fusion and IVD replacement $(5,6)$. However, to date, there is no effective method available to repair IVDD (7). Consequently, it is necessary to explore the pivotal pathogenesis of IVDD and identify available treatment methods.

Although various factors are relevant to the occurrence of IVDD, nutritional dysregulation is regarded to be the ultimate common pathway of IVDD, since the IVD is the largest non-vascular organ in the human body $(8,9)$. The material exchange between the IVD and the vertebral body mainly relies on the cranial and caudal endplates (EPs) $(10,11)$, which contain bone marrow contact channels (12). Studies have demonstrated that the nutrition acquisition of IVD is dependent on EP permeability and that there is a significant association between the permeability of the EP and the porosity of the bone marrow contact channel $(13,14)$. The calcification of the cartilaginous EP leads to the obstruction of the bone marrow contact channel, which may induce and accelerate the occurrence of IVDD; thus, the alteration of EPs is a critical feature of the physiopathology of IVDD (15).

Type IX collagen (Col9) is a heterogeneous collagen composed of three different $\alpha$ chains ( $\alpha 1, \alpha 2$ and $\alpha 3)$, which is 
predominantly expressed in cartilage $(16,17)$. It is also a major component of IVDs, vertebral EPs and developing vertebral bodies $(18,19)$. As a fiber-associated collagen with interrupted triple helices, Col9 is assembled with type II collagen ( $\mathrm{Col} 2)$ to form heterogeneous fibers. It is crosslinked with $\mathrm{Col} 2$ located on the surface of fibrils and its non-collagenous NC4 domain projecting out (20). Numerous interactions with other cartilage matrix proteins have been allocated to this domain, indicating that Col9 functions as an adaptor between the collagenous network and other extracellular matrix (ECM) superstructures (21). The Col9 polymorphism has been reported to be a risk factor for the occurrence of IVDD, particularly with advancing age. As one of the branches of Col9, previous studies have suggested that the $\alpha 2$ chain of $\mathrm{Col} 9$ (Col9a2) is closely associated with degenerative lumbar spinal stenosis and spondylolisthesis $(22,23)$.

However, it remains to be determined whether the deletion of Col9 $\alpha 2$ causes IVDD primarily by affecting EPs. Based on these observations, it was hypothesized that the absence of Col9 $\alpha 2$ may lead to IVDD through the modic change in EPs and other tissues in IVDs. Therefore, the present study examined the effects of Col9a2 knockout on EP structure, ECM deposition, matrix degradation and chondrocyte hypertrophy-related gene and protein expression alterations in IVD tissues.

\section{Materials and methods}

Animals. Mice with a deficiency in the Col9a2 gene (Col9a2 $\left.2^{-/}\right)$ were provided by the Nanjing Biomedical Research Institute of Nanjing University. The DNA from tail biopsies of mice was genotyped using PCR. All mice were housed at a constant room temperature of $20 \pm 2^{\circ} \mathrm{C}$ and humidity of $50-60 \%$ with a 12-h light/dark cycle and free access to water and standard food. A total of 36 male mice at 4 (12-15 g body weight), 8 (20-23 g body weight) and 12 (26-29 g body weight) weeks of age were used for further analysis. At each time-point, the Col9a $2^{-/}$group was compared with the wild-type (WT) control group ( $n=6$ per group). All mice were humanely euthanized via intraperitoneal injection of pentobarbital sodium $(150 \mathrm{mg} / \mathrm{kg})$. Animal death was confirmed by observation of cessation of heartbeat and respiration. The present study was approved by the Ethics Committee of Zhejiang Chinese Medical University (Hangzhou, China; approval no. 20190401-10) according to the National Institutes of Health Guide for the Care and Use of Laboratory Animals.

Micro- $C T(\mu C T)$. The lower thoracic and whole lumbar vertebrae of mice were fixed in $4 \%$ neutral formaldehyde solution at $4^{\circ} \mathrm{C}$ for $48 \mathrm{~h}$, transferred to $70 \%$ anhydrous ethanol and then scanned and examined using a high-resolution $\mu \mathrm{CT}$ scanner (Skyscan 1176; Bruker) with the following settings: Isotropic voxel size resolution, $9 \mu \mathrm{m}$; energy, $45 \mathrm{kV}$; current, $500 \mu \mathrm{A}$; and integration time, $780 \mathrm{msec}$. The lower thoracic ribs were included for the identification of L4-L5 IVD localization. Image reconstruction and analysis was performed using NRecon v1.6 (Bruker) and CTAn v1.9 (Bruker), respectively, followed by analysis of the L4-L5 IVD parameters using three-dimensional model visualization software (CTVolx, v3.0; Bruker). The IVD volume was defined by the region of
Table I. Primer sequences for quantitative PCR.

Gene/primer

direction

Sequence

\begin{tabular}{ll}
\hline $\begin{array}{l}\beta \text {-actin } \\
\text { Forward }\end{array}$ & 5'-GGAGATTACTGCCCTGGCTCCTA-3' \\
Reverse & 5'-GACTCATCTACTCCTGCTTGCTG-3' \\
Col2a1 & \\
Forward & 5'-TGGTCCTCTGGGCATCTCAGGC-3' \\
Reverse & 5'-GGTGAACCTGCTGTTGCCCTCA-3' \\
Mmp13 & \\
Forward & 5'-TTTGAGAACACGGGGAAGA-3' \\
Reverse & 5'-ACTTTGTTGCCAATTCCAGG-3' \\
Aggrecan & \\
Forward & 5'-CGCCACTTTCATGACCGAGA-3' \\
Reverse & 5'-TCATTCAGACCGATCCACTGGTAG-3' \\
Adamts5 & \\
Forward & 5'-CCAAATGCACTTCAGCCACGATCA-3' \\
Reverse & 5'-AATGTCAAGTTGCACTGCTGGGTG-3' \\
Col10a1 & \\
Forward & 5'-ACCCCAAGGACCTAAAGGAA-3' \\
Reverse & 5'-CCCCAGGATACCCTGTTTTT-3' \\
Runx2 & \\
Forward & 5'-GAGGGCACAAGTTCTATCTGGA-3' \\
Reverse & 5'-GGTGGTCCGCGATGATCTC-3' \\
\hline
\end{tabular}

Co19 $\alpha 2, \alpha 2$ chain of type IX collagen; Adamts5, ADAM metallopeptidase with thrombospondin type 1 motif 5; Runx2, Runx family transcription factor 2 .

interest (ROI) to cover the entire invisible space between the L4-L5 vertebrae and the cartilage EP volume was defined as covering the visible bone plate close to the vertebrae. A total of 50 consecutive ROI images were used to display the three-dimensional reconstruction of the EP and IVD space. The average of the volume and the height of the IVD were calculated.

Histochemistry and histomorphometry. The specimens were fixed in neutral formaldehyde solution at a volume fraction of $4 \%(0.1 \mathrm{~mol} / 1, \mathrm{pH} 7.4)$ for 3 days and then decalcified in $10 \%$ EDTA (buffered with pure water, $\mathrm{pH}$ 7.4) for 2 weeks. The samples were embedded in paraffin, cut into $3 \mu \mathrm{m}$-thick sections and stained with Alcian blue hematoxylin/orange $\mathrm{G}(\mathrm{ABH} / \mathrm{OG})$ and safranin $\mathrm{O}$ and fast green. A total of six different mice from each group at each time-point were selected for the assessment of EP, annulus fibrosus (AF) and nucleus pulposus (NP) using a light microscope (Carl Zeiss AG). The EP score was obtained as previously described and used to evaluate the degeneration of the EP, including structural disorganization, clefts and bony sclerosis $(24,25)$.

Immunohistochemistry (IHC) and immunofluorescence $(I F)$. IHC was performed using a standard protocol. In brief, 3- $\mu$ m-thick paraffin-embedded sections were incubated at 
A

B
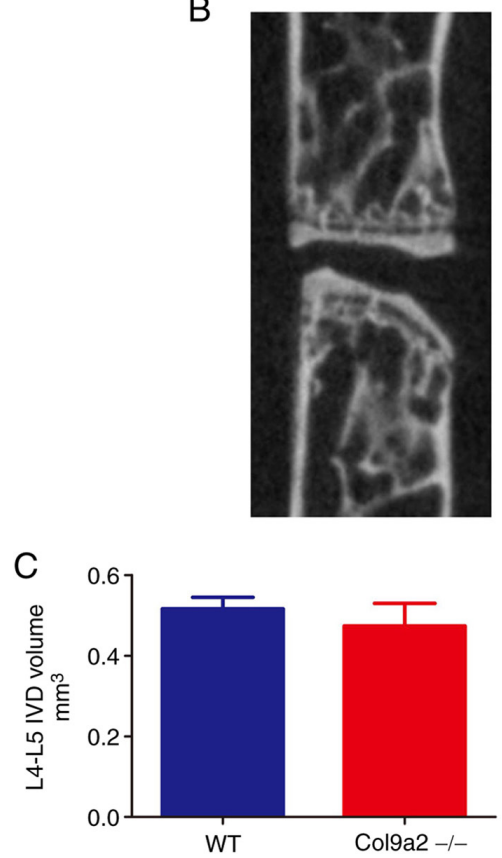
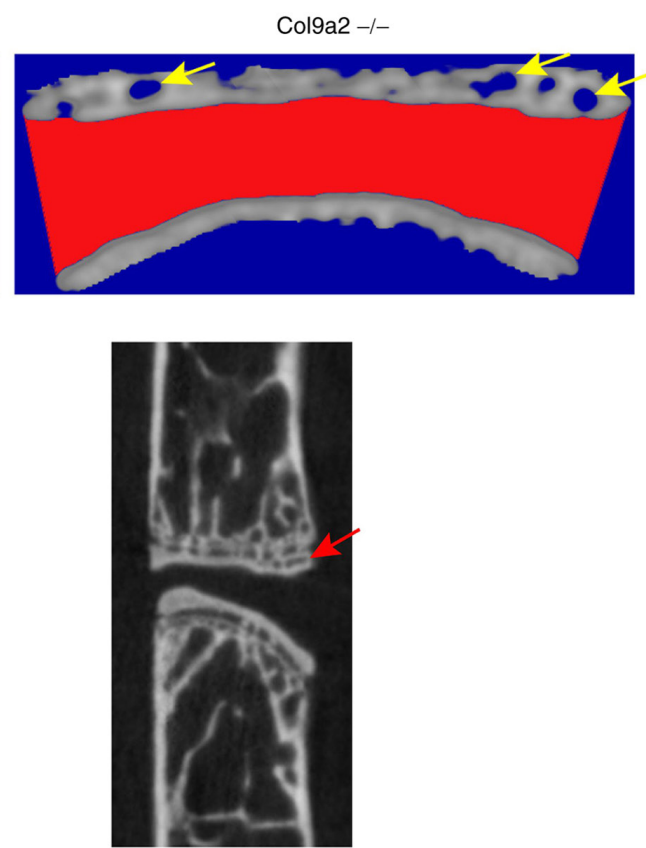

D

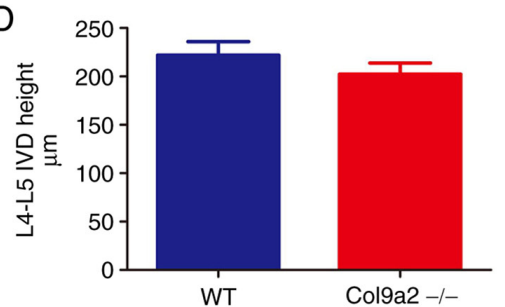

Figure 1. Measurement of microstructural parameters of EPs and changes in the volume and height of L4-L5 IVD by $\mu$ CT. (A) The three-dimensional images of EPs in the two groups of mice. Yellow arrows indicate the ossified area and the IVD space is indicated by the red color. (B) Mid-sagittal L4-L5 $\mu$ CT images of WT and Col9a2 $2^{-/}$mice. Calcified cavities are indicated by a red arrow. (C and D) Quantification of (C) L4-L5 IVD volume and (D) height. Mice were analyzed at 12 weeks ( $=6$ per group). Values are expressed as the mean \pm standard deviation. WT, wild-type; EPs, endplates; IVD, intervertebral disc; Col9 $\alpha 2, \alpha 2$ chain of type IX collagen.

$60^{\circ} \mathrm{C}$ for $4 \mathrm{~h}$ prior to deparaffinizing and dehydrating. The sections were then incubated in citrate buffer $(0.01 \mathrm{M}, \mathrm{pH} 6.0$; Beijing Solarbio Science \& Technology Co., Ltd.) at $60^{\circ} \mathrm{C}$ for $4 \mathrm{~h}$, or in pepsinum (OriGene Technologies, Inc.) at $37^{\circ} \mathrm{C}$ for $30 \mathrm{~min}$ for antigen retrieval. The samples were then treated with endogenous peroxidase blocker (cat. no. PV-6001; OriGene Technologies, Inc.) for $10 \mathrm{~min}$ and incubated with $0.3 \%$ Triton X-100 for $15 \mathrm{~min}$ at room temperature. The sections were then incubated with primary antibodies against Col9a2 (diluted 1:100; cat. no. sc398130; Santa Cruz Biotechnology, Inc.), Col2al (diluted 1:1,000 for IHC and 1:50 for IF; cat. no. ab34712; Abcam), Aggrecan (diluted 1:200 for IHC and 1:50 for IF; cat. no. NB100-74350; Novus Biologicals, LLC), Mmp13 (diluted 1:200; cat. no. ab39012; Abcam), ADAM metallopeptidase with thrombospondin type 1 motif 5 (Adamts5; diluted 1:200; cat. no. ab182795; Abcam), Col10a1 (diluted 1:200; cat. no. ab58632; Abcam) and Runx family transcription factor 2 (Runx2; diluted 1:300; cat. no. ab76956; Abcam) overnight at $4^{\circ} \mathrm{C}$. For IHC staining, the sections were incubated with a secondary goat anti-mouse/rabbit antibody (diluted 1:1,000; cat. no. 31234; Invitrogen; Thermo Fisher Scientific, Inc.) for $20 \mathrm{~min}$ at room temperature. Positive staining of sections was visualized using diaminobenzidine solution (Invitrogen; Thermo Fisher Scientific, Inc.), while hematoxylin was used for counterstaining. For the IF assay, the slides were incubated with fluorophore-conjugated secondary antibodies at room temperature for $30 \mathrm{~min}$ in the dark. The sections were counterstained with DAPI and observed under a fluorescence microscope (Carl Zeiss AG). A total of five images from each section were analyzed using Image-Pro Plus 6.0 (Media Cybernetics, Inc.). The area of Col2a1 or Col10a1-positive staining was calculated, while the Aggrecan-, Mmp13-, Adamts5- or Runx2-positive cells were obtained by counting the number of positively stained cells.

Reverse transcription-quantitative PCR (RT-qPCR). The L4-L5 segments of the spinal cords were removed from 12-week-old mice and the soft tissues were excised. Total RNA was extracted using the Qiagen RNeasy Mini kit (Qiagen $\mathrm{GmbH}$ ) and then reverse transcribed into cDNA using the RevertAid First Strand cDNA Synthesis kit (Invitrogen; Thermo Fisher Scientific, Inc.) according to manufacturers' protocols. As per the manufacturer's instructions, qPCR was performed for target genes using SYBR Premix Ex Taq ${ }^{\mathrm{TM}}$ II (Takara Biotechnology Co., Ltd.) with a QuantStudio ${ }^{\mathrm{TM}} 7$ Flex Real-Time PCR System (Thermo Fisher Scientific, Inc.). The thermocycling conditions were: Pre-denaturation at $94^{\circ} \mathrm{C}$ for $5 \mathrm{~min}$; followed by 40 cycles of denaturation at $94^{\circ} \mathrm{C}$ for $30 \mathrm{sec}$, 
A
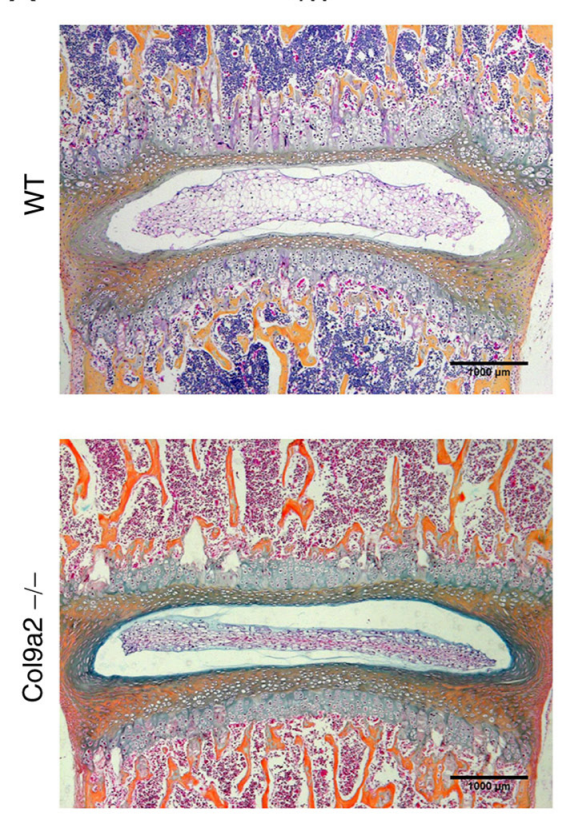

$8 \mathrm{~W}$
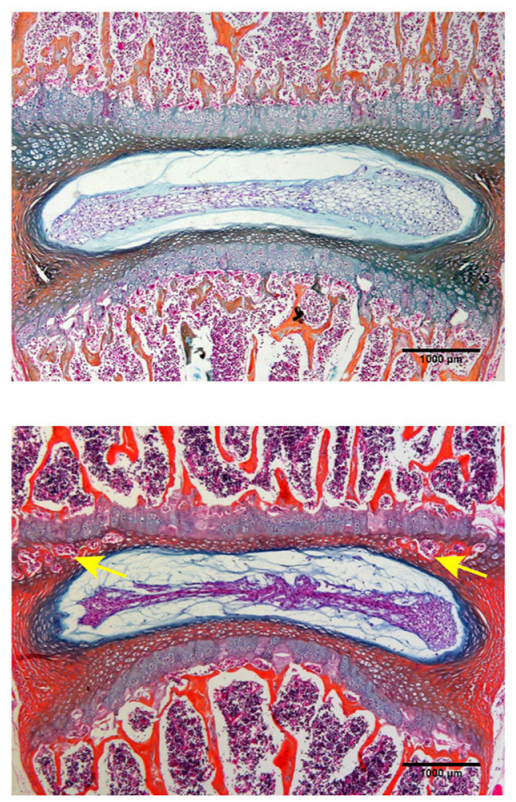

$12 \mathrm{~W}$
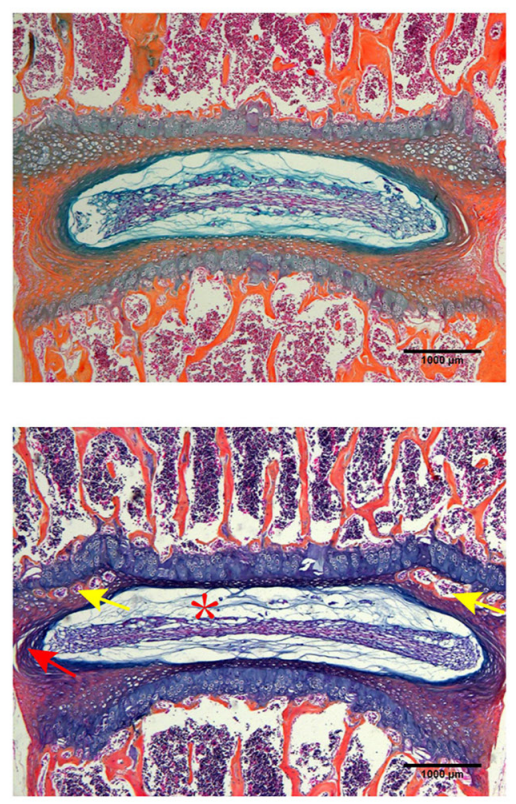

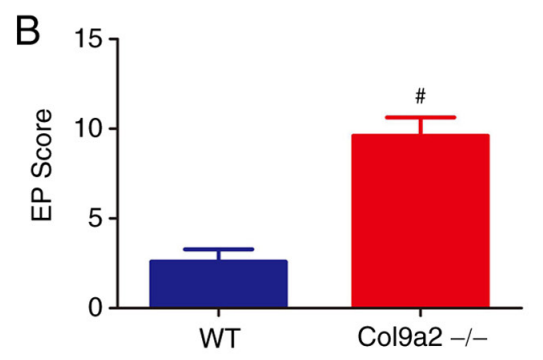

Figure 2. Accelerated osteochondral remodeling in EPs of Col9a2-/ mice. (A) Representative ABH/OG staining images of L4-L5 IVD in 4-, 8- and 12-week-old mice (scale bars, $1,000 \mu \mathrm{m}$ ). (B) EP score in Col9a $2^{-/}$or WT mice as an indication of EP degeneration. Yellow arrows indicate the ossific endplate, the red asterisk indicates the decrease of notochord cells and proteoglycans in the NP and the red arrow indicates the formation of cracks in the AF. Values are expressed as the mean \pm standard deviation ( $\mathrm{n}=6$ per group). ${ }^{*} \mathrm{P}<0.05$. WT, wild-type; $\mathrm{W}$, weeks; EP, endplate; NP, nucleus pulposus; AF, annulus fibrosus; ABH/OG, Alcian blue-hematoxylin/orange G; IVD, intervertebral disc; Col9 $\alpha 2, \alpha 2$ chain of type IX collagen.

annealing at $60^{\circ} \mathrm{C}$ for $30 \mathrm{sec}$ and extension at $72^{\circ} \mathrm{C}$ for $30 \mathrm{sec}$. The primer sequences are presented in Table I and the relative mRNA expression was measured using the $2^{-\Delta \Delta \mathrm{Cq}}$ method (26).

Statistical analysis. Statistical analysis was performed using SPSS software (version 25.0; IBM Corp). Values are expressed as the mean \pm the standard error of the mean and analyzed using a one-way ANOVA followed by Bonferroni's post-hoc test as appropriate. $\mathrm{P}<0.05$ was considered to indicate a statistically significant difference.

\section{Results}

Changes in EP and increases in porosity lead to early-stage IVDD in Col9a $2^{-/}$mice. $\mu \mathrm{CT}$ scanning was implemented to qualitatively estimate the lumbar spine of the Col9a $2^{-/}$and WT mice at 12 weeks of age. The cavities within the EPs were significantly increased in the CT three-dimensional images of the L4-L5 IVD (Fig. 1A), as well as in the median sagittal images of the CT scan (Fig. 1B). Although no statistically significant differences were observed, the IVD volume and height of Col9a2 $2^{--}$mice were reduced compared with those of WT mice (Fig. 1C and D), as determined through the analysis of the three-dimensional reconstruction images of the L4-L5 IVD. Thus, the CT data suggested that although there were no significant differences in the L4-L5 IVD volume and height between the Col9a2 $2^{-/-}$and WT mice, the ossification of the EPs was slightly increased and this is a phenotype of early-stage IVDD (27).

Osteochondral remodeling of the EP induces IVDD in $\mathrm{Col}_{9} \mathrm{a}^{-/-}$mice. Histochemistry and histomorphometry were performed to further observe the phenotypes of IVDD induced by $\mathrm{Col} 9 \mathrm{a} 2$ gene knockout. $\mathrm{ABH} / \mathrm{OG}$ staining revealed that there were no obvious histological differences between the two groups at 4 and 8 weeks, except that the 8-week-old $\mathrm{Col} 9 \mathrm{a}^{--/}$mice exhibited a slight calcification of the EPs. The EPs of the Col9a2 $2^{--}$mice began to undergo significant degeneration at 12 weeks of age compared with the WT mice, as indicated by the apparent calcification and ossification formation of the EPs (Fig. 2A). Furthermore, oteoglycan loss was also present in the NP of $\mathrm{Col} 9 \mathrm{a}^{-/-}$mice, as evidenced by less $\mathrm{ABH} / \mathrm{OG}$ and safranin $\mathrm{O}$ and fast green staining compared with the WT mice (Figs. 3 and 4). Furthermore, a large number of clefts was observed in the AF of the Col9a $2^{-/-}$mice with collagen dislocation and cytopenia, and even AF rupture 
WT
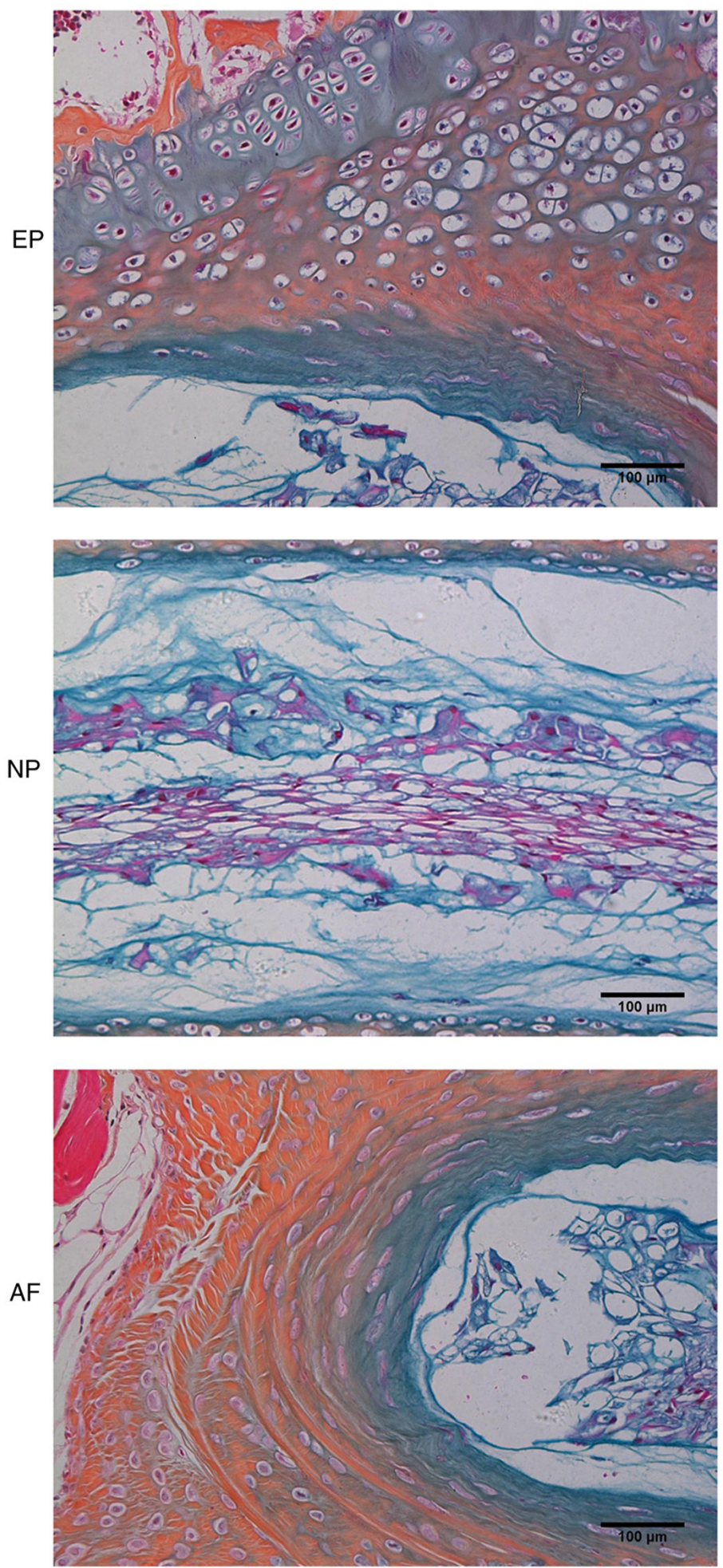

Col9a2 -1-
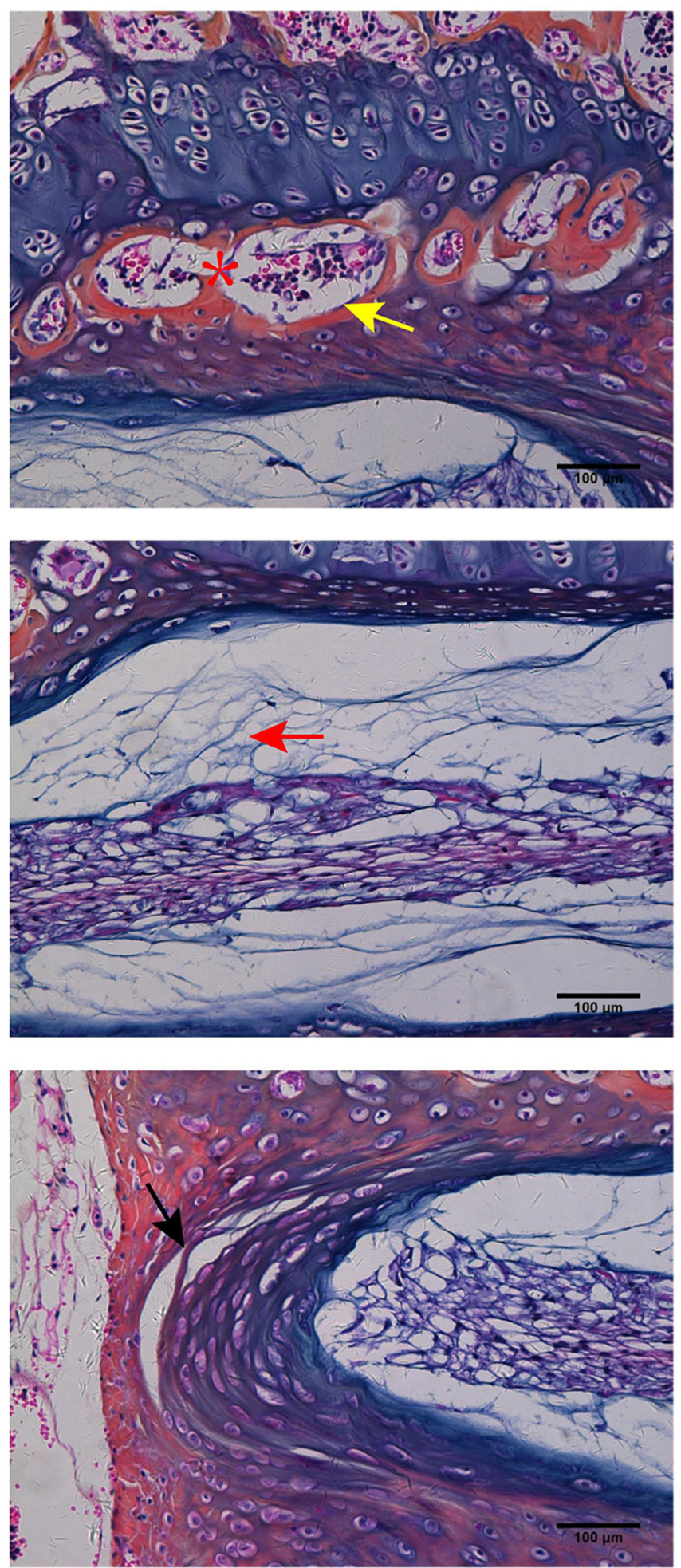

Figure 3. Representative higher-magnification ABH/OG staining images of L4-L5 IVD of 12-week-old mice compared to Fig. 2. Higher-magnification images of the EP, NP and AF (scale bars, $100 \mu \mathrm{m}$ ). Ossific nodules (yellow arrow) in the EP along with thickening of bony EP (red asterisk) were observed in the $\mathrm{Co} 19 \mathrm{a} 2^{--}$mice. Furthermore, a reduction of notochord cells and proteoglycan in the NP (red arrow) as indicated by a paucity of ABH/OG staining and crack formation within the $\mathrm{AF}$ (black arrow) were observed in the Col9a $2^{--}$mice ( $\mathrm{n}=6$ per group). WT, wild-type; EP, endplate; NP, nucleus pulposus; AF, annulus fibrosus; ABH/OG, Alcian blue-hematoxylin/orange G; IVD, intervertebral disc; Col $9 \alpha 2, \alpha 2$ chain of type IX collagen.

(Figs. 3 and 4B). The EP score, a histological evaluation of EP degeneration, was used to assess pathological changes, such as the degree of osteosclerosis, structural disorders and neovascularization. Of note, it was indicated that the EP score was significantly increased in the Col9a $2^{--}$mice compared with the WT mice at 12 weeks, confirming EP degeneration (Fig. 2B). The results of the histochemical and histomorphometric analysis indicated that the 12-week-old Col9a2 $2^{-/}$mice exhibited certain other manifestations 
A
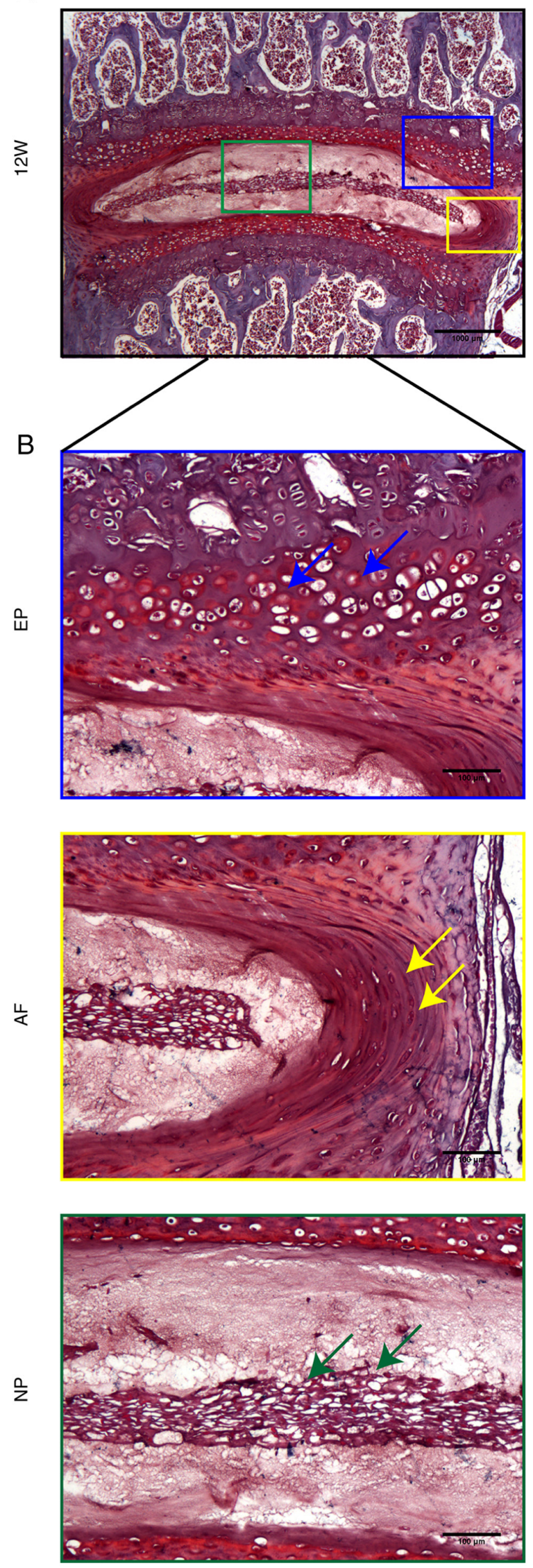

Col9a2 -1-
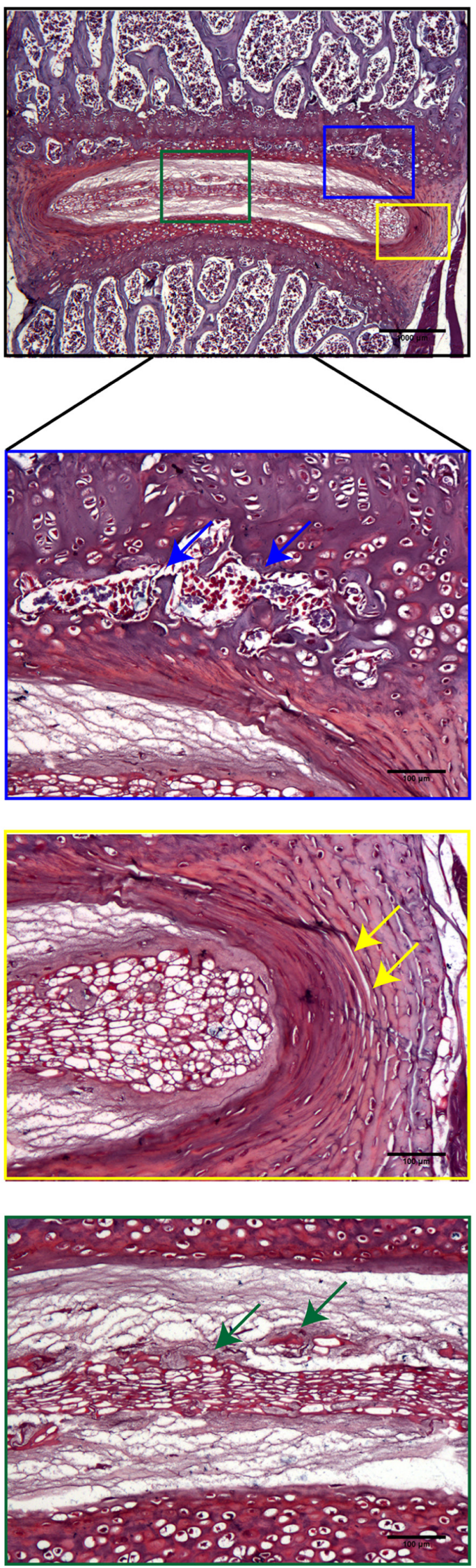

Figure 4. Safranin O and fast green staining images of L4-L5 IVD of 12-week-old mice. (A) Representative Safranin O and fast green staining images of L4-L5 IVD of 12-week-old WT and Col9a2 $2^{-/}$mice (scale bars, 1,000 $\mu \mathrm{m}$ ). (B) Higher-magnification images of the EP, NP and AF (scale bars, $100 \mu \mathrm{m}$ ). Blue arrows indicated ossific EP, yellow arrows indicate the formation of cracks in the AF and green arrows indicate the decrease of notochord cells and proteoglycans in the NP ( $\mathrm{n}=6$ per group). WT, wild-type; EP, endplate; NP, nucleus pulposus; AF, annulus fibrosus; IVD, intervertebral disc; Col9 $\alpha 2, \alpha 2$ chain of type IX collagen; W, weeks. 
A
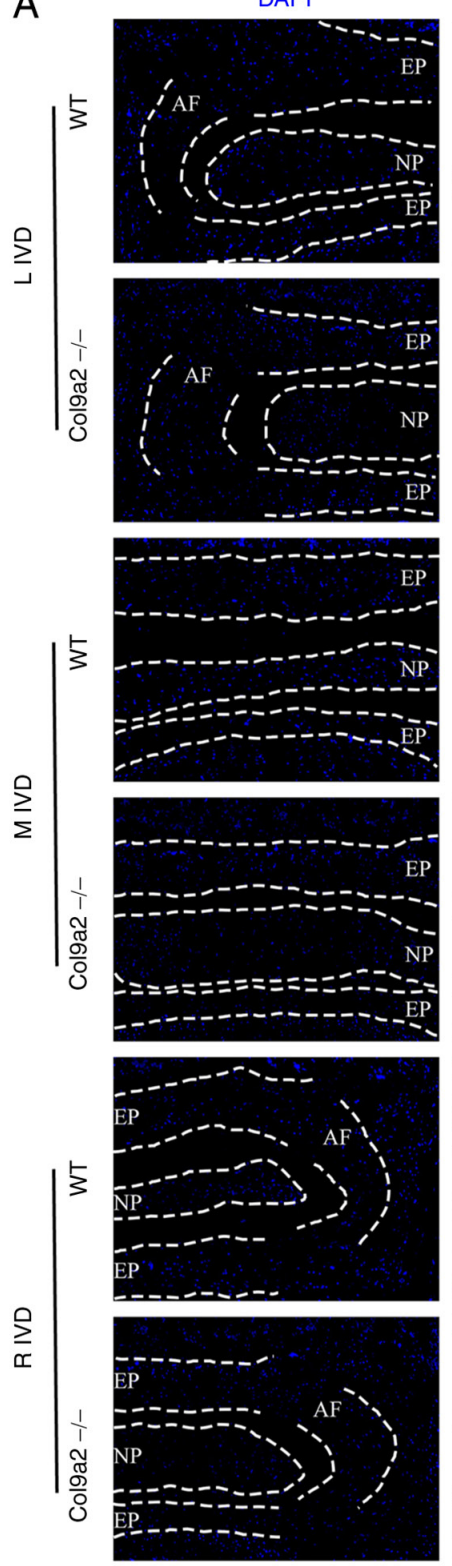

Col9a2
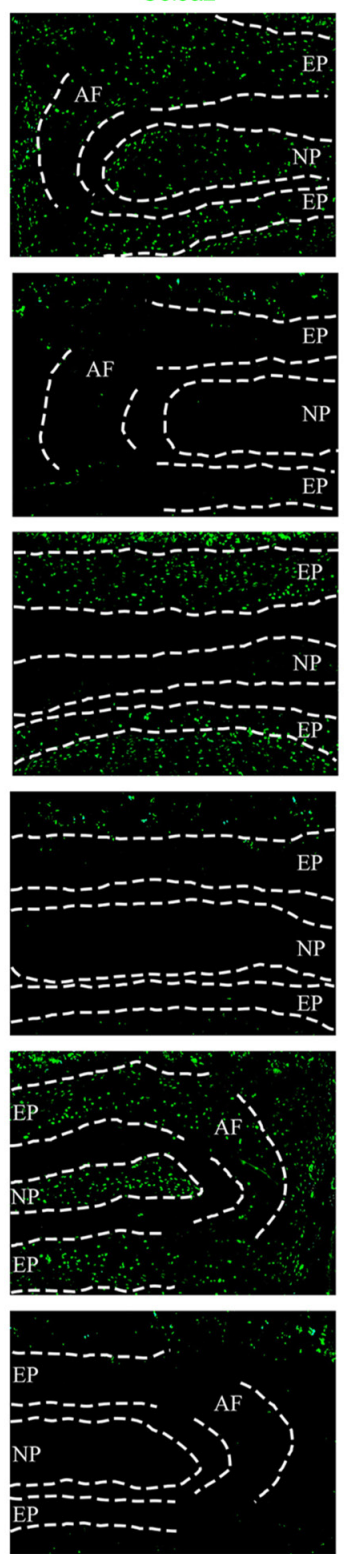

Merged
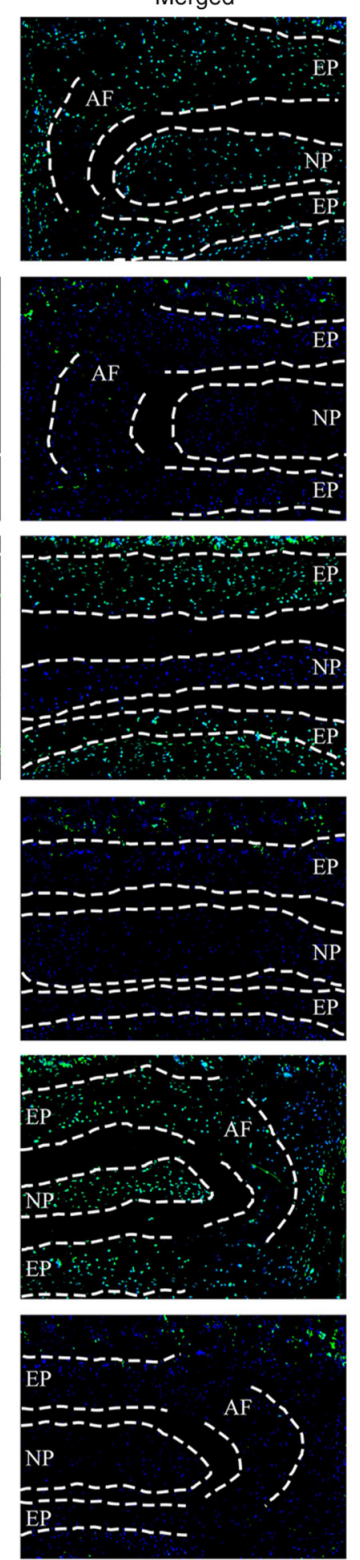

$B$
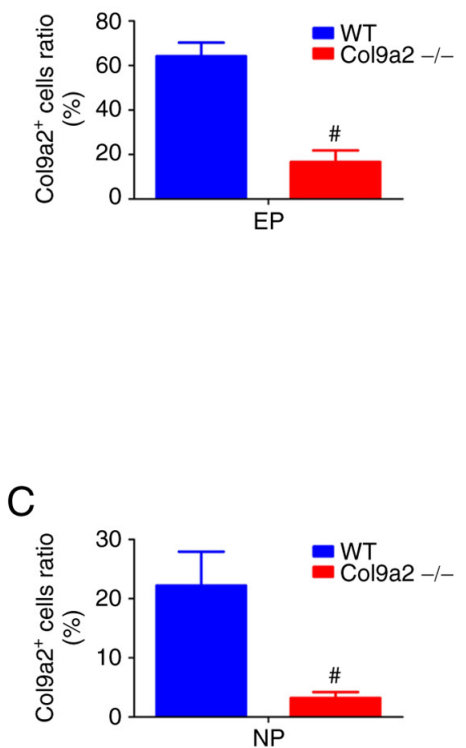

D

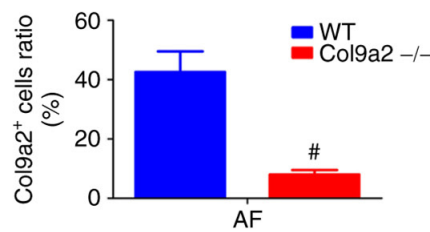

Figure 5. Col9a2 immunofluorescence staining of IVDs of 2-week-old mice. (A) Col9a2 immunofluorescence staining of EP, NP and AF. Nuclei were stained blue with DAPI and Col9a2 expression was detected as green (magnification, x200). (B-D) Quantification of Co9a2 in the (B) EP, (C) NP and (D) AF, further

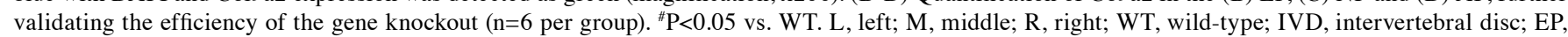
endplate; NP, nucleus pulposus; $\mathrm{AF}$, annulus fibrosus; $\mathrm{Col} 9 \alpha 2, \alpha 2$ chain of type IX collagen.

of IVDD, such as AF rupture and proteoglycan reduction, in addition to distinct EP degeneration, relative to WT mice.

\section{Reduced expression of ECM deposition-associated proteins} and increased matrix degradation- and chondrocyte hypertrophy-related protein levels in Col9a $2^{-/-}$mice. Further experiments were performed to determine whether the protein levels of IVD-related proteins in $\mathrm{Col} 9 \mathrm{a} 2^{-/-}$mice were also altered. IF was used to locate the expression of Col9a2 and verify the efficiency of gene knockout (Fig. 5A). Col9a2 was extensively expressed in the IVDs of WT mice, while its expression was low in the $\mathrm{Col} 9 \mathrm{a}^{-/}$mice (Fig. 5B-D). IHC and IF analysis were then performed to assess the expression levels of Col2a1, Aggrecan, Mmp13, Adamts5, Col10a1 and Runx2 in the IVDs. The expression levels of Col2a1 and Aggrecan, which indicate ECM deposition, were markedly decreased in the IVDs of Col9a2 $2^{--}$mice as compared with those in WT mice (Figs. 6 and 7). Furthermore, chondrocyte matrix damage, as assessed by staining for Mmp13 and Adamts5 in the EPs and AF of Col9a $2^{--}$mice, increased significantly compared with that in the WT mice (Fig. 8). Furthermore, chondrocyte hypertrophy, as evaluated by the number of Col10a1- and Runx2-positive cells, was uniformly distributed in the EPs and AF of WT mice, whereas in the EPs and outer layer of AF of Col9a $2^{-1-}$ mice, positively stained cells were observed at sites where the chondrocytes merged together; however, positive cells were not present in the larger EP cavities and the expression levels of Col10a1 and Runx 2 were markedly increased in the IVDs of Col9a $2^{-1-}$ mice compared with those in WT mice (Fig. 9). These results suggested that the absence of the Col9a2 gene decreased 
A
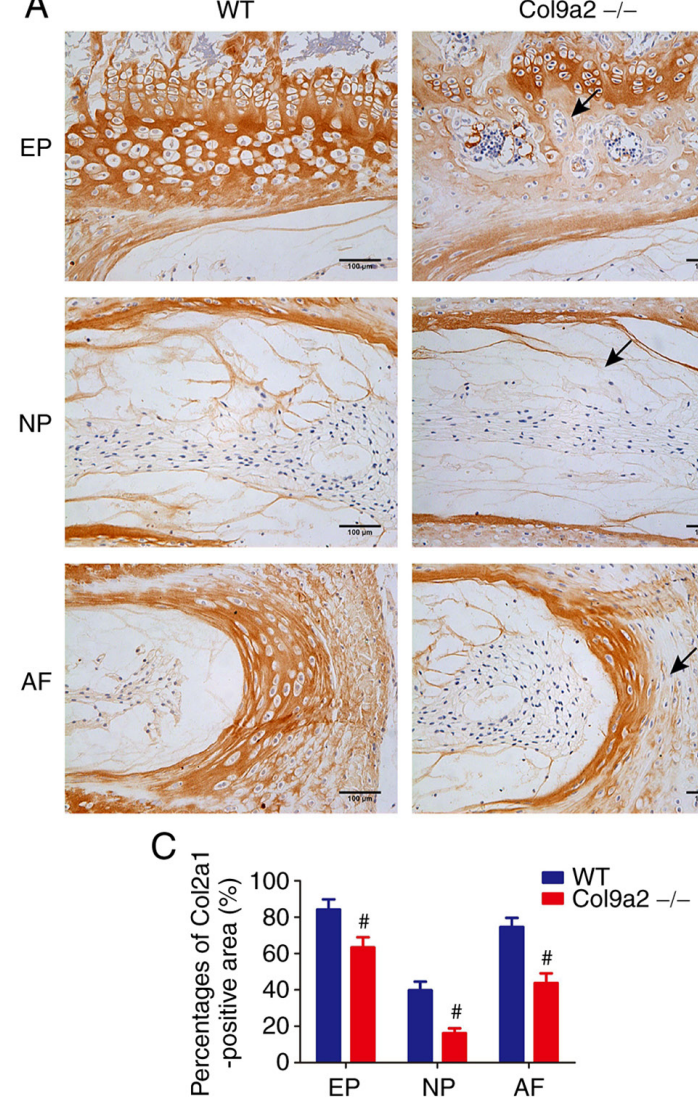

Col9a2 -1-
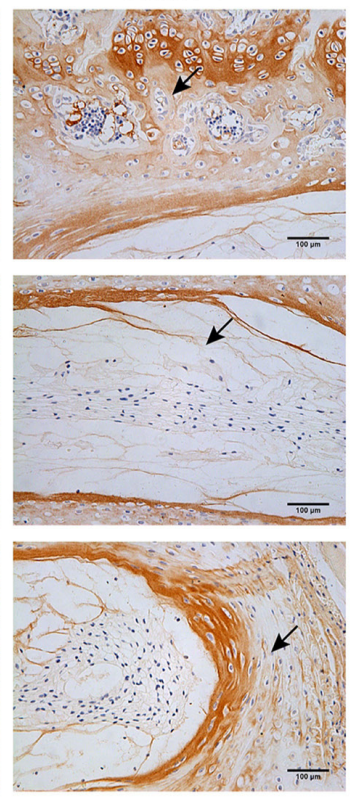

B
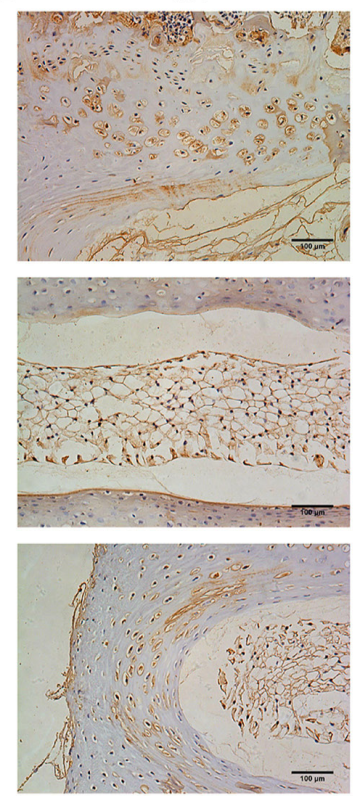

D 듀

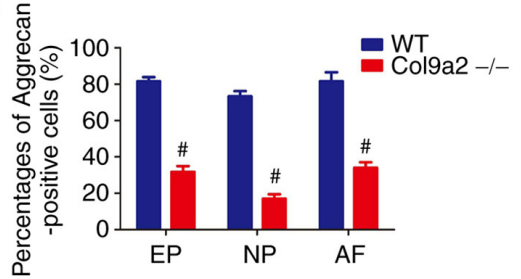

Figure 6. Immunohistochemical detection of Col2a1 and Aggrecan. (A and B) Representative images (scale bars, $100 \mu \mathrm{m}$ ). Positive immunostaining was noted as brown staining. Black arrows indicate loss of (A) Col2a1 and (B) Aggrecan in Col9a2 ${ }^{-/}$mice compared with the WT mice, with the most pronounced reduction of Col2a1 in the ossification zone of the cartilage EP and the outer layer of AF and a significant decrease of Aggrecan in the NP. Quantification of (C) Col2a1 and (D) Aggrecan in the EP, NP and AF. Values are expressed as the mean \pm standard deviation (n=6 per group). ${ }^{\#} \mathrm{P}<0.05$ vs. WT. WT, wild-type; EP, endplate; NP, nucleus pulposus; AF, annulus fibrosus; Col9 $\alpha 2, \alpha 2$ chain of type IX collagen.

the expression levels of ECM deposition-associated proteins and increased that of matrix degradation- and chondrocyte hypertrophy-related proteins in IVD cartilage tissues, which exacerbated the occurrence and development of IVDD.

Diminished expression of genes associated with ECM deposition and enhanced expression of genes related to matrix degradation and chondrocyte hypertrophy in Col9a2 $2^{-/-}$mice. RT-qPCR was performed to further determine the mRNA expression levels of ECM deposition-, matrix degradationand chondrocyte hypertrophy-related genes in the IVD tissue of $\mathrm{Col} 9 \mathrm{a} 2^{-/-}$mice. As expected, the data demonstrated that the mRNA expression trends of Col2a1, Aggrecan, Mmp13, Adamts5, Col10a1 and Runx2 were similar to the results of protein expression described above: Knockout of the Col9a2 gene diminished the mRNA expression levels of Col2al and Aggrecan (Fig. 10A and B), while the mRNA expression levels of Mmp13, Adamts5, Col10a1 and Runx2 were increased (Fig. 10C-F). These results suggested that deletion of the Col9a2 gene suppressed ECM synthesis and accelerated matrix degradation and chondrocyte hypertrophy in the IVD tissue.

\section{Discussion}

Col9 is a component of the ECM of cartilage, which contributes to the integrity of the cartilage structure. Col9 92 is one of the branched chains of Col9 and its deletion has been indicated to be related to the early occurrence of IVDD. However, its specific mechanisms of action have remained to be further elucidated. The EP is a selective permeability barrier in IVDs and has attracted increasing attention in recent years. Nutritional access to the IVD is dependent on the EP. In addition, severe IVDD is more common in patients with EP modic changes, indicating that EP modic changes are related to the initiation and development of IVDD (28). In the present study, the histopathological staining results revealed that there was no significant difference between the two groups of mice at 4 or 8 weeks; however, the $\mathrm{Col}_{9} \mathrm{a}^{-/-}$mice at 12 weeks exhibited the manifestations of early-stage IVDD, which was characterized by EP calcification cavities, reduced proteoglycan content and AF rupture, amongst other signs. EP degradation is considered to begin with abnormal calcification (15). Calcium crystalline salts are deposited into the pores of the EP, resulting in a decrease in EP permeability. During the aging process, the calcified EP undergoes ossification and sclerosis, which is eventually replaced by bone $(29,30)$. This process is considered to reduce the transport of nutrients from the vertebral marrow to the IVD. Thus, EP remodeling caused by Col9a2 gene knockout may lead to increased permeability, which may be the most important factor contributing to IVDD.

Furthermore, changes in the ECM composition of EP chondrocytes have an important role in the occurrence 
A
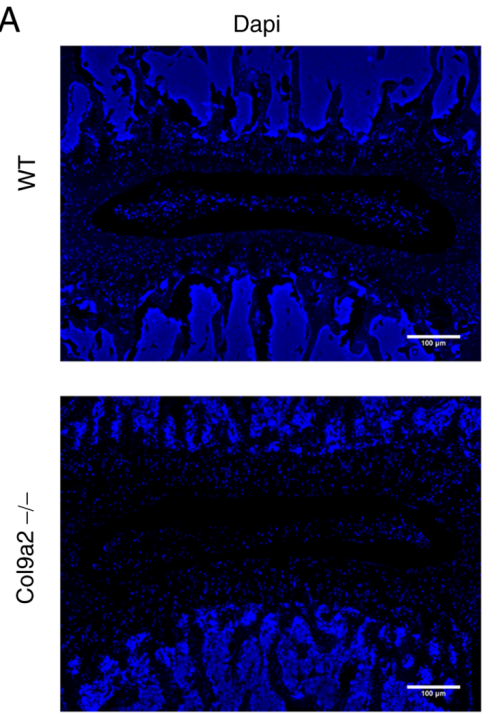

B
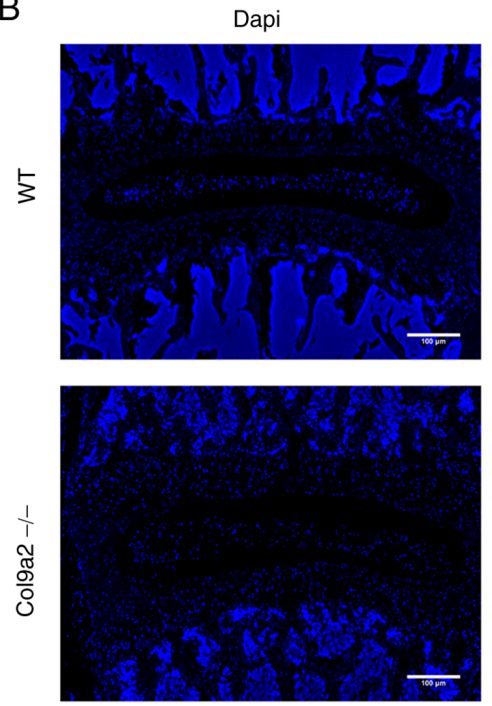

C

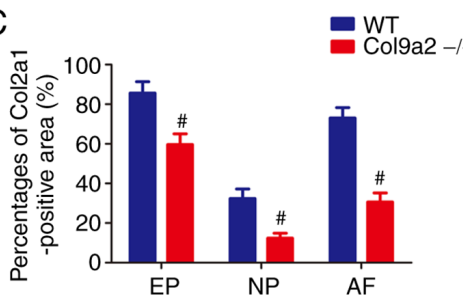

Col2a1
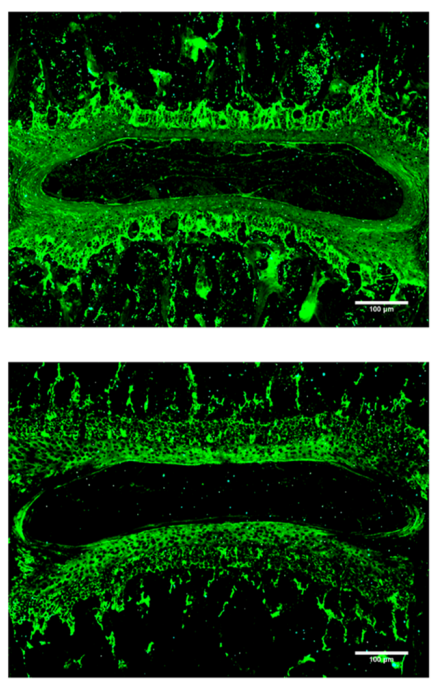

Aggrecan
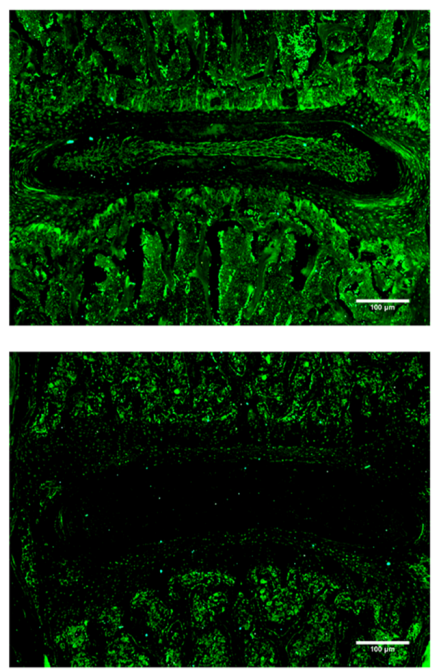
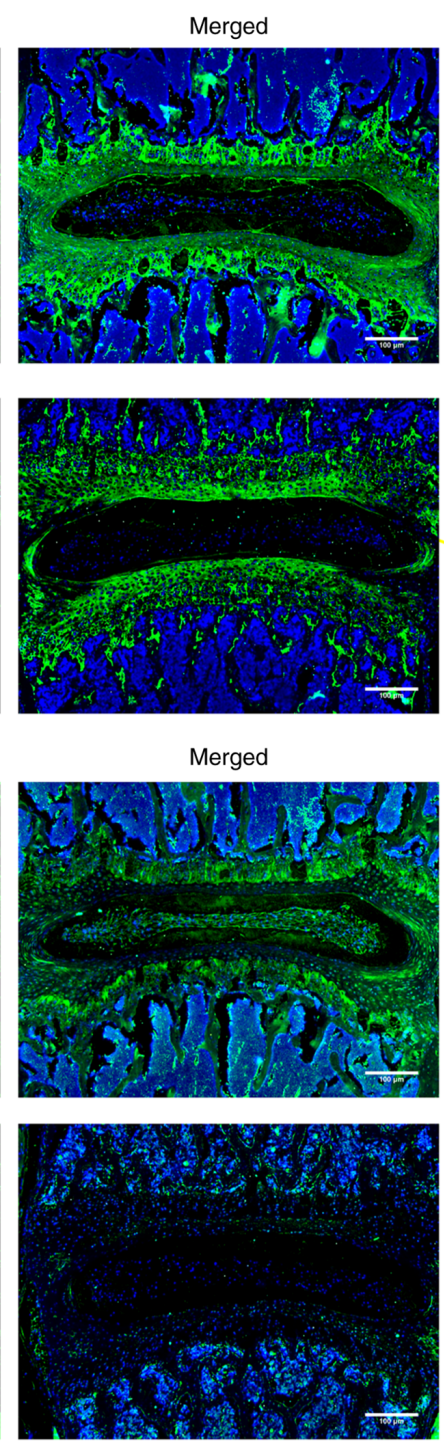

D

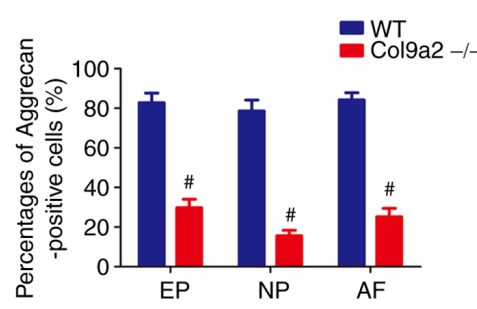

Figure 7. Immunofluorescence detection of extracellular matrix-related protein of L4-L5 IVD of 12-week-old mice. (A and B) Representative images of immunofluorescence of (A) Col2a1 and (B) Aggrecan (scale bars, $100 \mu \mathrm{m}$ ). Nuclei were stained blue with DAPI, while Col2a1 and Aggrecan expression was detected as green. (C and D) Quantification of (C) Col2al and (D) Aggrecan in the EP, NP and AF. Values are expressed as the mean \pm standard deviation (n=6 per group). "P<0.05 vs. WT. WT, wild-type; EP, endplate; NP, nucleus pulposus; AF, annulus fibrosus; Col9 $\alpha 2, \alpha 2$ chain of type IX collagen.

and development of IVDD. Studies have demonstrated that EPs are critical to the nutritional supply of IVD due to the limited blood supply $(31,32)$. EP cartilage is a thin layer of hyaline cartilage located between the vertebrae and IVD, mainly composed of chondrocytes and ECM. EP chondrocytes are responsible for the maintenance and circulation of cartilage-specific ECM molecules. The major components of ECM within the IVDs are Col2al and Aggrecan, which endow EP cartilage with weight-bearing properties and nutrient exchange abilities $(33,34)$. Therefore, their contents are essential for the proper function of the IVDs, particularly in the cartilaginous EP. The excessive destruction of ECM, and in particular, the loss of $\mathrm{Col} 2 \mathrm{al}$ and Aggrecan, may be risk factors for IVDD (35). Furthermore, Mmps and Adamts are the main enzymes that degrade Col2al and Aggrecan, while Mmp13 and Adamts5 are the hallmarks of cartilage degeneration (36). There is substantial and increasing evidence to indicate that Mmp13 and Adamts5 expression is upregulated in IVD tissues and cells, and are closely associated with the process of ECM rupture and disc degeneration (37). In the present study, the results of the IHC and RT-qPCR analysis revealed a distinct decrease in Col2a1 and Aggrecan protein 
A
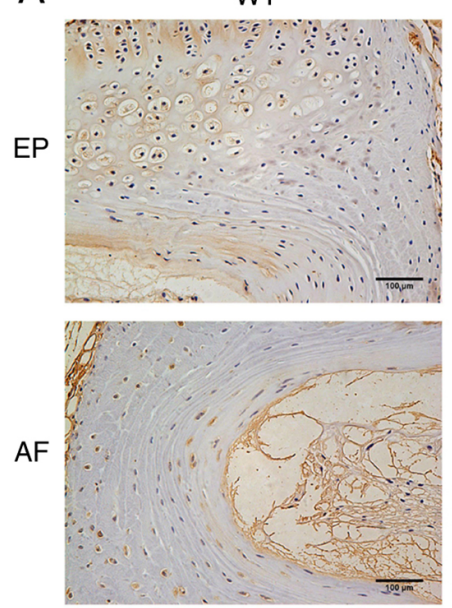

$\mathrm{C}^{\mathrm{m}}$

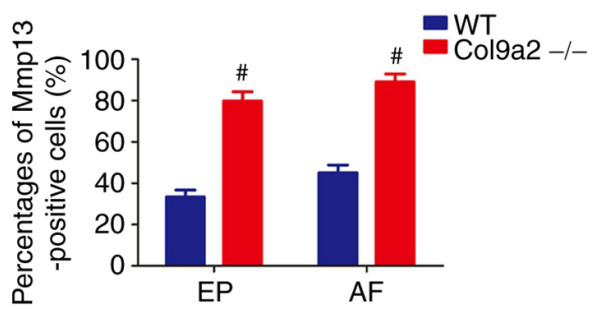

B
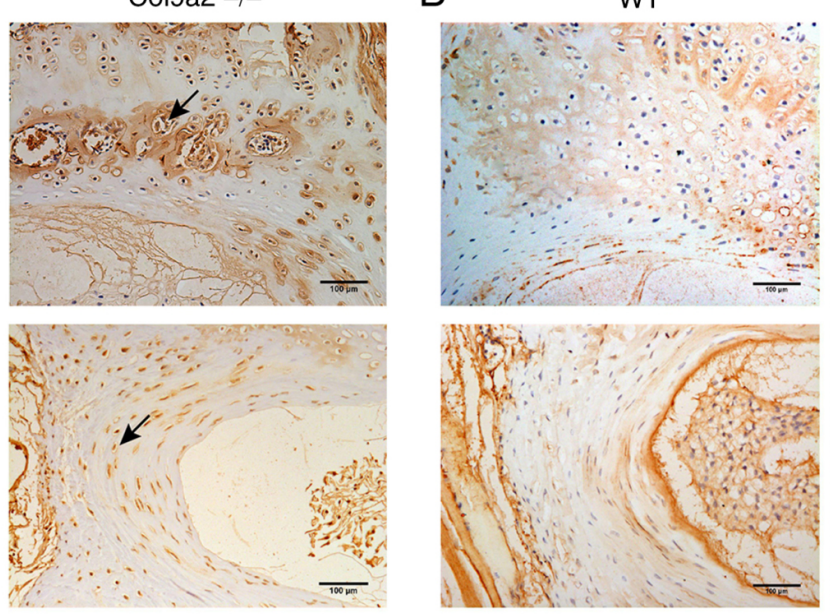

D
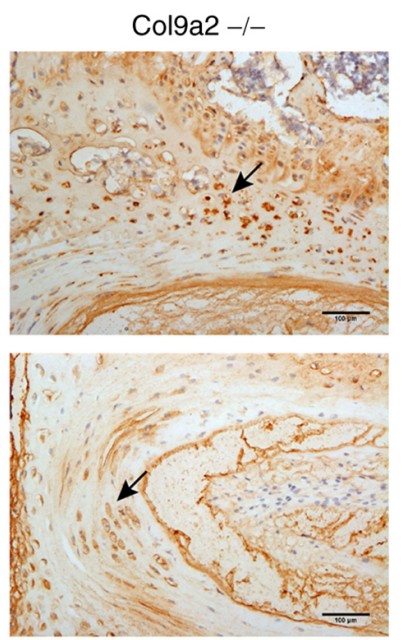

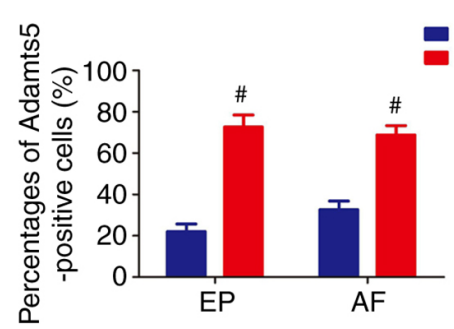

Figure 8. Immunohistochemical detection of Mmp13 and Adamts5. (A and B) Representative immunohistochemistry images. Positive immunostaining was noted as brown staining (scale bars, $100 \mu \mathrm{m}$ ). Black arrows indicate increased expression of (A) Mmp13 and (B) Adamts5 in Col9a2 ${ }^{-/-}$mice compared with the WT mice, with the most pronounced increase in Mmp13 expression in the calcified basal region of the EP and the entire AF, and the expression of Adamts5 was markedly increased in both the EP and AF. Quantification of (C) Mmp13 and (D) Adamts5 in the EP and AF. Values are expressed as the mean \pm standard deviation ( $\mathrm{n}=6$ per group). ${ }^{\text {} P}<0.05$ vs. WT. WT, wild-type; EP, endplate; AF, annulus fibrosus; Col9 $\alpha 2, \alpha 2$ chain of type IX collagen; Adamts5, ADAM metallopeptidase with thrombospondin type 1 motif 5.

A
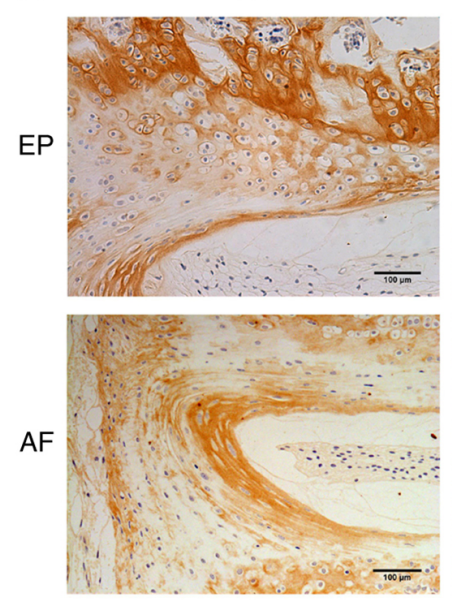

C

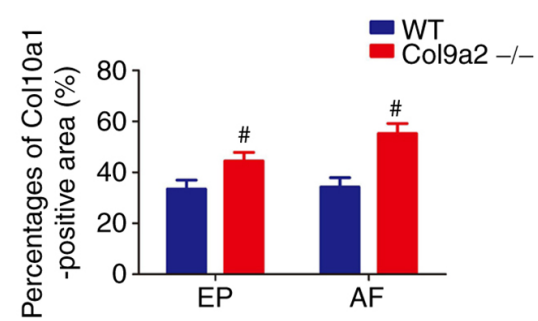

B
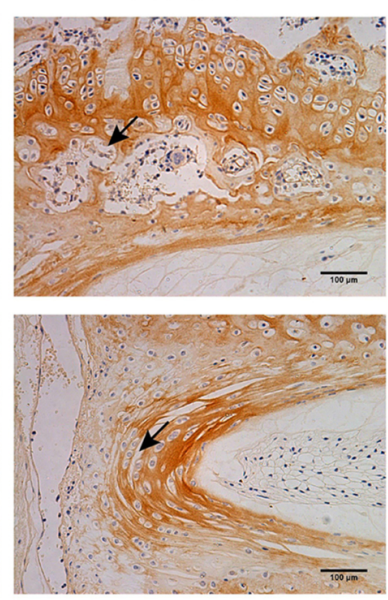

ETT
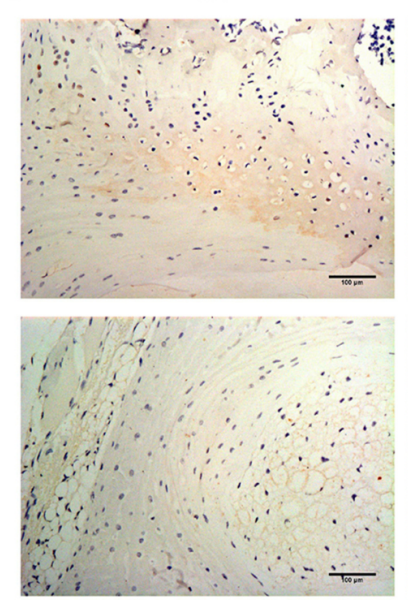

WT Col9a2 -1ang
ith the
mants5
metal-

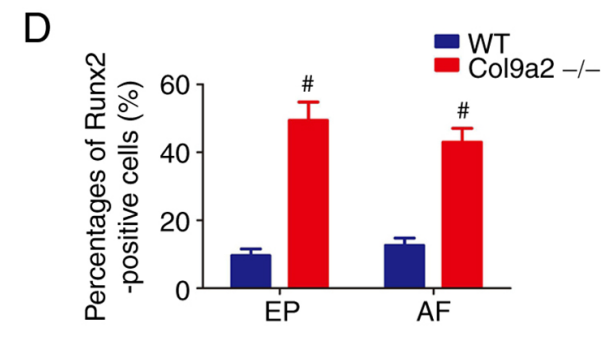

Figure 9. Immunohistochemical analysis of Col10a1 and Runx2. (A and B) Representative immunohistochemistry images. Positive immunostaining was observed as brown staining (scale bars, $100 \mu \mathrm{m}$ ). Black arrows indicate increased expression of (A) Col10a1 and (B) Runx 2 in Col9a $2^{-/-}$mice compared with WT mice, with the most distinct enhancement of Col10a1 in the outer layer of AF, while Runx 2 expression was markedly increased in both the EP and AF. (C and D) Quantification of (C) Col10a1 and (D) Runx2 in the EP and AF. Values are expressed as the mean \pm standard deviation ( $\mathrm{n}=6$ per group). "P<0.05 vs. WT. WT, wild-type; EP, endplate; AF, annulus fibrosus; Col9 $\alpha 2, \alpha 2$ chain of type IX collagen; Runx 2 , Runx family transcription factor 2 . 
A

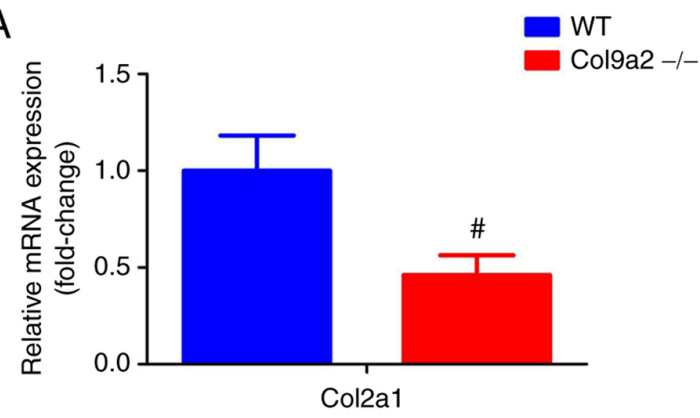

C

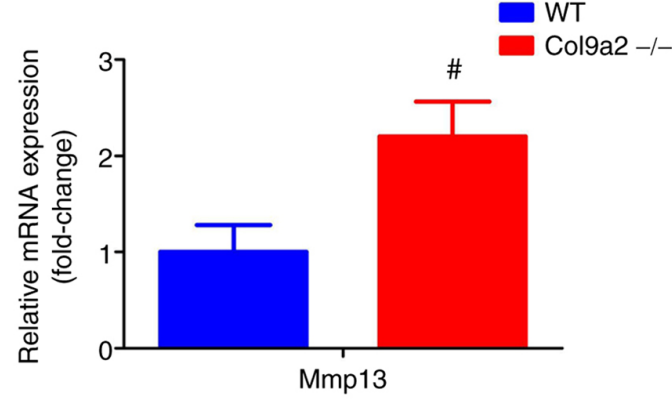

E

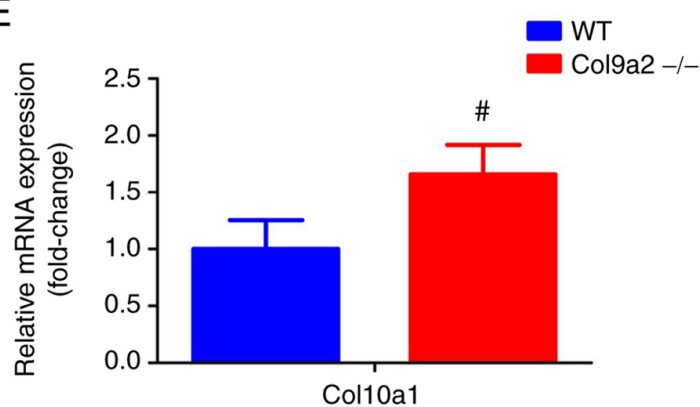

$B$

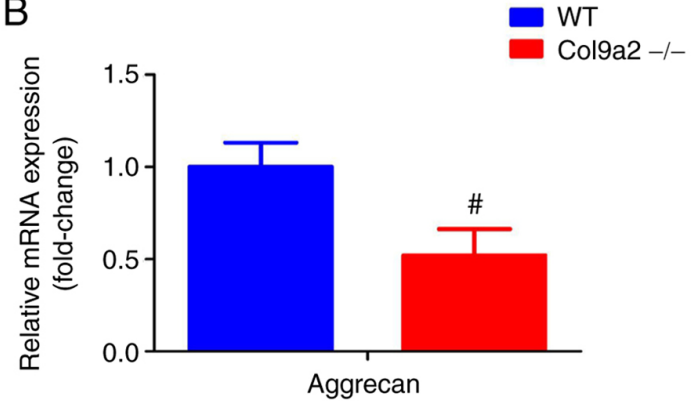

D

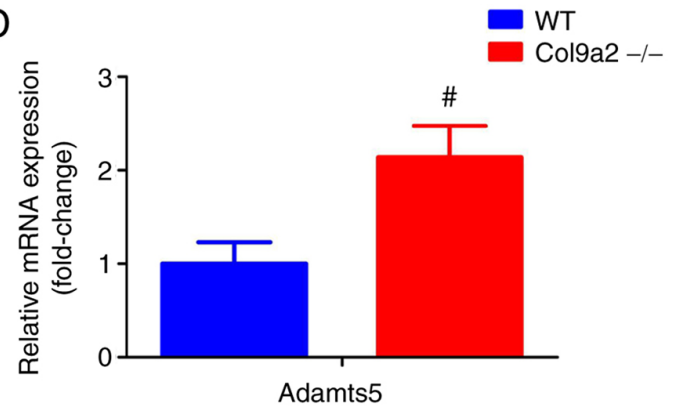

$\mathrm{F}$

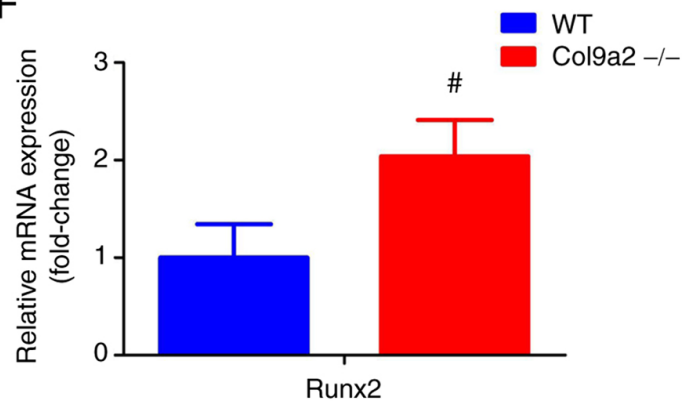

Figure 10. mRNA expression levels of Col2a1, Aggrecan, Mmp13, Adamts5, Col10a1 and Runx2. Quantitative results of mRNA expression analysis of (A) Col2a1, (B) Aggrecan, (C) Mmp13, (D) Adamts5, (E) Col10a1 and (F) Runx2. Values are expressed as the mean \pm standard deviation (n=6 per group). ${ }^{\#} \mathrm{P}<0.05$ vs. WT. WT, wild-type; Col9 $\alpha 2, \alpha 2$ chain of type IX collagen; Runx2, Runx family transcription factor 2; Adamts5, ADAM metallopeptidase with thrombospondin type 1 motif 5 .

and mRNA levels, while the levels of Mmp13 and Adamts5 were markedly increased in Col9a $2^{-/-}$mice compared with WT mice. This suggests that Col9a2 gene knockout causes excessive ECM destruction and insufficient ECM synthesis, which further exacerbates EP calcification and ultimately accelerates the progression of IVDD.

The PI3K/Akt signaling pathway has been reported to be a key regulator of terminal chondrocyte differentiation in embryonic and adult chondrogenesis $(38,39)$, and increased PI3K and Akt phosphorylation may enhance the levels of Mmp13 and Adamts5, but decrease the levels of Col2a1 and Aggrecan, ultimately exacerbating the process of IVDD (40). Furthermore, as a marker of chondrocyte hypertrophy, the precise function of Col10a1 remains to be determined; however, it is generally accepted that there is an association between Col10a1 synthesis and endochondral osteogenesis (41). Furthermore, Runx2, as a marker of chondrocyte terminal differentiation, may alter the phenotypic state of chondrocytes (42). In addition, it has been indicated that the expression of Col10a1 and Runx2 in EP chondrocytes is associated with degenerative disc disease $(43,44)$. It has been demonstrated that the ECM-receptor interaction signaling pathway is dysregulated in the early stages of IVDD (40). As components of the ECM, Col9a2, Col2a1 and Aggrecan have important roles in the ECM-receptor interaction signaling pathway. IVDD caused by Col9a2 gene knockout may be related to the imbalance of the ECM-receptor interaction signaling pathway.

In conclusion, the present study provided insight into the deteriorating effects of Col9a2 gene knockout on IVDD, clearly demonstrating that Col9a2 gene knockout induces and accelerates the progression of IVDD. The underlying mechanisms may be related to the dysregulation of the PI3K/Akt and ECM-receptor interaction signaling pathways due to the deletion of the Col9a2 gene, resulting in decreased protein and mRNA expression levels of Col2al and Aggrecan, and increased expression levels of Mmp13, Adamts5, Col10a1 and Runx2. This leads to enhanced EP calcification, which subsequently disrupts the structural integrity and function of the EP, and ultimately exacerbates the process of IVDD. These changes may be a plausible explanation for IVDD associated with the absence of the Col9a2 gene. However, the research is still at a preliminary stage, and thus, the results require to be further confirmed by clinical trials or other animal experiments. The exact molecular mechanisms of 
IVDD and Col9a2 gene deletion warrant further investigation.

\section{Acknowledgements}

Not applicable.

\section{Funding}

This research was funded by the Chinese National Natural Science Foundation (grant nos. 81774346, 81873324, 81973869, 81904221 and 81904219), the Traditional Chinese Medical Administration of Zhejiang Province (grant nos. 2021ZZ014, 2018ZA034, 2019ZQ018 and 2018ZZ011), the Health Commission of Zhejiang Province (grant no. 2019RC225) and the Shaoxing Science and Technology Project (grant no. 2018C30114).

\section{Availability of data and materials}

The datasets used and/or analyzed during the current study are available from the corresponding author on reasonable request.

\section{Authors' contributions}

PT, HJ and CW made substantial contributions to the conception and design of the study. HX, RD, QZ, LF and QG performed the experiments. HX, RD, QZ, CX, PZ, SL and ZZ interpreted the data and drafted the manuscript. PW, JL, HR and $\mathrm{SH}$ performed the statistical analysis and reviewed the manuscript critically for important intellectual content. HX, RD and QZ confirm the authenticity of all the raw data. All authors read and approved the final manuscript.

\section{Ethics approval and informed consent}

All animal experiments were approved by the Institutional Ethics Committee of Zhejiang Chinese Medical University (Hangzhou, China; no. 20190401-10).

\section{Patient consent for publication}

Not applicable.

\section{Competing interests}

The authors declare that they have no competing interests.

\section{References}

1. Luoma K, Vehmas T, Kerttula L, Gronblad M and Rinne E: Chronic low back pain in relation to modic changes, bony endplate lesions, and disc degeneration in a prospective MRI study. Eur Spine J 25: 2873-2881, 2016.

2. Izzo R, Popolizio T, D'Aprile P and Muto M: Spinal pain. Eur J Radiol 84: 746-756, 2015.

3. Cazzanelli P and Wuertz-Kozak K: MicroRNAs in intervertebral disc degeneration, apoptosis, inflammation, and mechanobiology. Int J Mol Sci 21: 3601, 2020.

4. Frapin L, Clouet J, Delplace V, Fusellier M, Guicheux J and Le Visage C: Lessons learned from intervertebral disc pathophysiology to guide rational design of sequential delivery systems for therapeutic biological factors. Adv Drug Deliv Rev 149-150: 49-71, 2019.
5. Chen BL, Guo JB, Zhang HW, Zhang YJ, Zhu Y, Zhang J, Hu HY, Zheng YL and Wang XQ: Surgical versus non-operative treatment for lumbar disc herniation: A systematic review and meta-analysis. Clin Rehabil 32: 146-160, 2018.

6. Wu PH, Kim HS and Jang IT: Intervertebral disc diseases PART 2: A review of the current diagnostic and treatment strategies for intervertebral disc disease. Int J Mol Sci 21: 2135 , 2020.

7. Makanji H, Schoenfeld AJ, Bhalla A and Bono CM: Critical analysis of trends in lumbar fusion for degenerative disorders revisited: Influence of technique on fusion rate and clinical outcomes. Eur Spine J 27: 1868-1876, 2018.

8. Shapiro IM, Vresilovic EJ and Risbud MV: Is the spinal motion segment a diarthrodial polyaxial joint: What a nice nucleus like you doing in a joint like this? Bone 50: 771-776, 2012.

9. Chen S, Fu P, Wu H and Pei M: Meniscus, articular cartilage and nucleus pulposus: A comparative review of cartilage-like tissues in anatomy, development and function. Cell Tissue Res 370: 53-70, 2017.

10. Kang R, Li H, Ringgaard S, Rickers K, Sun H, Chen M, Xie L and Bünger $\mathrm{C}$ : Interference in the endplate nutritional pathway causes intervertebral disc degeneration in an immature porcine model. Int Orthop 38: 1011-1017, 2014.

11. Tomaszewski KA, Adamek D, Konopka T, Tomaszewska R and Walocha JA: Endplate calcification and cervical intervertebral disc degeneration: The role of endplate marrow contact channel occlusion. Folia Morphol (Warsz) 74: 84-92, 2015.

12. Mattei TA: Osteoporosis delays intervertebral disc degeneration by increasing intradiscal diffusive transport of nutrients through both mechanical and vascular pathophysiological pathways. Med Hypotheses 80: 582-586, 2013.

13. Laffosse JM, Accadbled F, Molinier F, Bonnevialle N, de Gauzy JS and Swider P: Correlations between effective permeability and marrow contact channels surface of vertebral endplates. J Orthop Res 28: 1229-1234, 2010.

14. Rodriguez AG, Slichter CK, Acosta FL, Rodriguez-Soto AE, Burghardt AJ, Majumdar S and Lotz JC: Human disc nucleus properties and vertebral endplate permeability. Spine (Phila $\mathrm{Pa}$ 1976) 36: 512-520, 2011.

15. Han Y, Li X, Yan M, Yang M, Wang S, Pan J, Li L and Tan J: Oxidative damage induces apoptosis and promotes calcification in disc cartilage endplate cell through ROS/MAPK/NF- $\kappa \mathrm{B}$ pathway: Implications for disc degeneration. Biochem Biophys Res Commun 516: 1026-1032, 2019.

16. Paassilta P, Pihlajamaa T, Annunen S, Brewton RG, Wood BM, Johnson CC, Liu J, Gong Y, Warman ML, Prockop DJ, et al: Complete sequence of the 23-kilobase human COL9A3 gene. Detection of Gly-X-Y triplet deletions that represent neutral variants. J Biol Chem 274: 22469-22475, 1999.

17. Pihlajamaa T, Vuoristo MM, Annunen S, Perala M, Prockop DJ and Ala-Kokko L: Human COL9A1 and COL9A2 genes. Two genes of 90 and $15 \mathrm{~kb}$ code for similar polypeptides of the same collagen molecule. Matrix Biol 17: 237-241, 1998.

18. Kamper M, Paulsson M and Zaucke F: Absence of collagen IX accelerates hypertrophic differentiation in the embryonic mouse spine through a disturbance of the Ihh-PTHrP feedback loop. Cell Tissue Res 367: 359-367, 2017.

19. Kamper M, Hamann N, Prein C, Clausen-Schaumann H, Farkas Z, Aszodi A, Niehoff A, Paulsson M and Zaucke F: Early changes in morphology, bone mineral density and matrix composition of vertebrae lead to disc degeneration in aged collagen IX -/- mice. Matrix Biol 49: 132-143, 2016.

20. Eyre DR, Pietka T, Weis MA and Wu JJ: Covalent cross-linking of the NC1 domain of collagen type IX to collagen type II in cartilage. J Biol Chem 279: 2568-2574, 2004.

21. Bruckner P: Suprastructures of extracellular matrices: Paradigms of functions controlled by aggregates rather than molecules. Cell Tissue Res 339: 7-18, 2010.

22. Noponen-Hietala N, Kyllonen E, Mannikko M, Kyllönen E, Männikkö M, Ilkko E, Karppinen J, Ott J and Ala-Kokko L: Sequence variations in the collagen IX and XI genes are associated with degenerative lumbar spinal stenosis. Ann Rheum Dis 62: 1208-1214, 2003.

23. Hyun SJ, Park BG, Rhim SC, Bae CW, Lee JK, Roh SW and Jeon SR: A haplotype at the COL9A2 gene locus contributes to the genetic risk for lumbar spinal stenosis in the Korean population. Spine (Phila Pa 1976) 36: 1273-1278, 2011. 
24. Boos N, Weissbach S, Rohrbach H, Weiler C, Spratt KF and Nerlich AG: Classification of age-related changes in lumbar intervertebral discs: 2002 volvo award in basic science. Spine (Phila Pa 1976) 27: 2631-2644, 2002.

25. Masuda K, Aota Y, Muehleman C, Imai Y, Okuma M, Thonar EJ, Andersson GB and An HS: A novel rabbit model of mild, reproducible disc degeneration by an anulus needle puncture: Correlation between the degree of disc injury and radiological and histological appearances of disc degeneration. Spine (Phila $\mathrm{Pa}$ 1976) 30: 5-14, 2005.

26. Livak KJ and Schmittgen TD: Analysis of relative gene expression data using real-time quantitative PCR and the 2(-Delta Delta $\mathrm{C}(\mathrm{T})$ ) method. Methods 25: 402-408, 2001

27. Ruiz Wills C, Foata B, Gonzalez Ballester MA, Karppinen J and Noailly J: Theoretical explorations generate new hypotheses about the role of the cartilage endplate in early intervertebral disk degeneration. Front Physiol 9: 1210, 2018.

28. Jiang C, Guo Q, Jin Y, Xu JJ, Sun ZM, Zhu DC, Lin JH, Tian NF, Sun LJ, Zhang XL and Wu YS: Inhibition of EZH2 ameliorates cartilage endplate degeneration and attenuates the progression of intervertebral disc degeneration via demethylation of Sox-9. EBioMedicine 48: 619-629, 2019.

29. Yuan FL, Xu RS, Ye JX, Zhao MD, Ren LJ and Li X: Apoptotic bodies from endplate chondrocytes enhance the oxidative stress-induced mineralization by regulating PPi metabolism. J Cell Mol Med 23: 3665-3675, 2019.

30. Dudli S, Ballatori A, Bay-Jensen AC, McCormick ZL, O'Neill CW, Demir-Deviren S, Krug R, Heggli I, Juengel A, Karppinen J, et al: Serum biomarkers for connective tissue and basement membrane remodeling are associated with vertebral endplate bone marrow lesions as seen on MRI (Modic changes). Int J Mol Sci 21: 3791, 2020.

31. Liu Z, Easson GW, Zhao J, Makki N, Ahituv N, Hilton MJ, Tang SY and Gray RS: Dysregulation of STAT3 signaling is associated with endplate-oriented herniations of the intervertebral disc in Adgrg6 mutant mice. PLoS Genet 15: e1008096, 2019.

32. Rade M, Määttä JH, Freidin MB, Airaksinen O, Karppinen J and Williams FM: Vertebral endplate defect as initiating factor in intervertebral disc degeneration: Strong association between endplate defect and disc degeneration in the general population. Spine (Phila Pa 1976) 43: 412-419, 2018.

33. Herrero CF, Garcia SB, Garcia LV and Aparecido Defino HL: Endplates changes related to age and vertebral segment. Biomed Res Int 2014: 545017, 2014.

34. Jackson AR, Huang CY and Gu WY: Effect of endplate calcification and mechanical deformation on the distribution of glucose in intervertebral disc: A 3D finite element study. Comput Methods Biomech Biomed Engin 14: 195-204, 2011.
35. Li X, Yang S, Han L, Mao K and Yang S: Ciliary IFT80 is essential for intervertebral disc development and maintenance. FASEB J 34: 6741-6756, 2020.

36. Zhang T, Dai Y, Zhang L, Tian Y, Li Z and Wang J: Effects of edible oils with different n-6/n-3 PUFA ratios on articular cartilage degeneration via regulating the NF- $\kappa \mathrm{B}$ signaling pathway. J Agric Food Chem 68: 12641-12650, 2020.

37. McCann MR, Veras MA, Yeung C, Lalli G, Patel P, Leitch KM, Holdsworth DW, Dixon SJ and Séguin CA: Whole-body vibration of mice induces progressive degeneration of intervertebral discs associated with increased expression of Il-1 $\beta$ and multiple matrix degrading enzymes. Osteoarthritis Cartilage 25: 779-789, 2017.

38. Kita K, Kimura T, Nakamura N, Yoshikawa H and Nakano T: $\mathrm{PI} 3 \mathrm{~K} /$ Akt signaling as a key regulatory pathway for chondrocyte terminal differentiation. Genes Cells 13: 839-850, 2008.

39. Yang S, Zhang F, Ma J and Ding W: Intervertebral disc ageing and degeneration: The antiapoptotic effect of oestrogen. Ageing Res Rev 57: 100978, 2020.

40. Zhang Q, Shen Y, Zhao S, Jiang Y, Zhou D and Zhang Y: Exosomes miR-15a promotes nucleus pulposus-mesenchymal stem cells chondrogenic differentiation by targeting MMP-3. Cell Signal 86: 110083, 2021.

41. Hristova GI, Jarzem P, Ouellet JA, Roughley PJ, Epure LM, Antoniou J and Mwale F: Calcification in human intervertebral disc degeneration and scoliosis. J Orthop Res 29: 1888-1895, 2011.

42. Sun MM and Beier F: Chondrocyte hypertrophy in skeletal development, growth, and disease. Birth Defects Res C Embryo Today 102: 74-82, 2014.

43. Zhang Q, Huang M, Wang X, Xu X, Ni M and Wang Y: Negative effects of ADAMTS-7 and ADAMTS-12 on endplate cartilage differentiation. J Orthop Res 30: 1238-1243, 2012.

44. Iwata M, Aikawa T, Hakozaki T, Arai K, Ochi H, Haro H, Tagawa M, Asou Y and Hara Y: Enhancement of Runx2 expression is potentially linked to $\beta$-catenin accumulation in canine intervertebral disc degeneration. J Cell Physiol 230: 180-190, 2015.

This work is licensed under a Creative Commons Attribution-NonCommercial-NoDerivatives 4.0 International (CC BY-NC-ND 4.0) License. 